\title{
Propuestas para construir un nuevo modelo de desarrollo contenidas en el Informe sobre Desarrollo Humano El Salvador 2010
}

William Pleitez

Coordinador y editor general del Informe sobre Desarrollo Humano El Salvador

\section{Introducción}

$\mathbf{L}^{2}$ a principal novedad del Informe sobre Desarrollo Humano El Salvador 2010 (IDHES 2010), denominado “De la pobreza y el consumismo al bienestar de la gente", es que contiene una diversidad de propuestas para la construcción de un nuevo modelo de desarrollo para el país basadas en el enfoque de desarrollo humano. Tales propuestas son el resultado de dos análisis complementarios: uno de carácter histórico, en el que se examinan los modelos de desarrollo y las políticas económicas y sociales practicadas en El Salvador desde los inicios de nación independiente; y el otro, de desarrollo comparado, que permite identificar qué hicieron otros países pequeños que en un momento de su historia fueron tan pobres como El Salvador, pero que ahora destacan entre los de mayor desarrollo humano.

II. La principal falencia de los modelos económicos ensayados en El Salvador.

El análisis histórico contenido en el Informe concluye que, contrariamente a lo que convencionalmente se cree, El Salvador desde mediados del siglo XIX ha venido practicando una suerte de "modelo económico único" con algunas variantes. Un modelo que ha privilegiado la apertura a los mercados internacionales por sobre la creación de una demanda interna; que ha concebido los bajos salarios de los trabajadores como la principal ventaja para competir internacionalmente; que ha dependido de un limitado número de productos de exportación; y que además ha elevado el dogmatismo y la polarización ideológica a patrones de la práctica política.

El modelo agroexportador se fundamentó en un intento por aprovechar los mercados internacionales. A la base estaba la concepción de los trabajadores como "mano de obra barata". No fue muy diferente el modelo de sustitución de importaciones, que le apostó más a la formación de capital del sector industrial que a la generación de capacidades en la gente. A finales de los años 80 se adoptó un modelo orientado inicialmente a la atracción de inversiones y la diversificación y aumento de la producción exportable, pero que terminó dependiendo de la "exportación" directa de la mano de obra y de las remesas enviadas por esta. 
Cada modelo, ciertamente, ha dejado sus huellas. Con el café se modificó la estructura de tenencia de la tierra, surgieron los primeros grupos empresariales locales y se consolidaron las bases de una economía agroexportadora. El modelo de industrialización por sustitución de importaciones abrió paso a un acelerado proceso de urbanización de la población, permitió un crecimiento importante de las clases medias y fortaleció las relaciones económicas con el resto de países centroamericanos. Finalmente, durante la vigencia del modelo actual se expandió la maquila, se aceleraron las migraciones laborales y se ha edificado una economía de consumo y servicios dependiente de las remesas familiares, que se han convertido en la principal variable macroeconómica del país.

Para romper ese molde, el país necesita poner luces altas y mirar hacia el pasado y vislumbrar un futuro diferente. Todo esfuerzo económico y social que se emprenda debe responder, y no en último término, a la pregunta clave: ¿qué papel se le otorga a la gente?

Diversos analistas - cuando examinan el lento crecimiento de la economía salvadoreña de nuestros días- parecen seguir ignorando el papel crucial que tiene la inversión en la gente, especialmente para países pequeños, que están obligados a buscar una inserción exitosa en los mercados mundiales. El listado de medidas que sugieren es extenso: mayor flexibilización laboral, más incentivos al turismo, apostarle a la exportación de productos nostálgicos, convertir al país en un centro logístico, intensificar las concesiones de activos del Estado a compañías privadas, etc. Curiosamente, en ninguna de estas apuestas se menciona a la gente.

Aceptar este tipo de recetas equivaldría a seguir poniendo "la carreta delante de los bueyes". Y no es que en apuestas como las mencionadas no haya oportunidades, pero la historia ha demostrado que serán otra promesa incumplida más, si no se fundamentan en apostarle al valor agregado derivado de invertir cada vez más en la gente.

La experiencia internacional ha probado que la mayoría de países pequeños que ahora son ricos le han apostado a desarrollar las capacidades de sus habitantes. El mejoramiento constante de esas habilidades ha pasado a convertirse en un objetivo central de sus políticas. De otra manera, este tipo de "recetas" económicas por muy lógicas que parezcan en el papel terminan empujando a la gente a buscarse el futuro lejos de su propio país, como es el caso de El Salvador. Las personas quieren progresar, vivir bien sus vidas y no aparecer como espectadores en una fiesta organizada por otros. Quieren aliados, no benefactores.

\section{Principales desafíos socioeconómicos de El Salvador de hoy}

Para dejar de ser un país subdesarrollado, en materia socioeconómica El Salvador deberá enfrentar exitosamente los siguientes desafíos:

3.1 Altas tasas de subutilización laboral. De cada cien personas que forman parte de la población económicamente activa (PEA) siete están desempleadas y 44 subempleadas.

3.2 Persistencia de elevados niveles de pobreza y déficits sociales. Alrededor de cuatro de cada diez hogares salvadoreños son pobres. La escolaridad promedio apenas alcanza 6 años, y la tasa de analfabetismo de las personas mayores de 10 años ó más es de 14\%. Entre los menores de 5 años, el 14\% presentan retardo en talla en las zonas urbanas, y $24 \%$ en el área rural.

3.3 Graves desequilibrios macroeconómicos. Durante los últimos años la situación macroeconómica del país se 
ha deteriorado sustancialmente, dando lugar a cuatro desequilibrios.

- El país consume más de lo que produce: en 2009, por cada $\$ 100$ que el país produjo, se consumieron $\$ 102.4$ (entre gasto de las familias y Gobierno). El Salvador ocupa la tercera posición en el mundo de los países con la mayor relación consumo/producto interno bruto (PIB).

- Bajos niveles de ahorro e inversión: El Salvador invierte poco y ahorra aún menos: en 2008 y 2009 la inversión promedió el $14 \%$ del PIB, y el ahorro el 9.3\%. En contraste, los países de alto crecimiento económico presentan tasas de ahorro e inversión en relación con el PIB de $25 \%$ o más).

- Déficit comercial creciente y modesta capacidad de atraer inversión extranjera directa (IED): el déficit comercial ha pasado de representar el 13\% del PIB en 1991 a más de $20 \%$ del PIB en los últimos años. Por su parte, los flujos de IED han sido bajos si se compara con otros países, y más aún si se les compara con su importancia relativa a las remesas: entre 1992 y 2008 la IED promedió \$350 millones por año, versus más de $\$ 1.930$ millones de remesas en el mismo período.

- Precaria situación fiscal: la deuda pública total como porcentaje del PIB ha aumentado en 20 puntos porcentuales en los últimos once años, pasando de 33\% en 1998 a 53\% en 2009.

3.4 Bajo crecimiento económico: en gran medida como consecuencia de los desequilibrios mencionados, El Salvador ha registrado una tasa de crecimiento per cápita promedio de apenas 1.1\% durante los últimos 50 años. Para el futuro, las perspectivas son similares, pues El Salvador se ubica entre los cuatro países de menor crecimiento proyectado para 2011 y 2012 de un total de 25 países de América Latina y el Caribe.

IV. Principios orientadores de las propuestas para un nuevo modelo de desarrollo

4.1 Bienestar. El desarrollo centrado en la gente busca ampliar las libertades reales de las personas, de manera que puedan llevar adelante los planes de vida que consideren valiosos.

4.2 Cohesión social. Se refiere a la necesidad de establecer consensos entre la ciudadanía, las fuerzas sociales, económicas y políticas y el gobierno basados en la justicia social, el respeto al estado de derecho y la primacía de los intereses generales sobre los intereses particulares. Las principales áreas en las que se requieren consensos son: la canalización del gasto público entre distintos sectores, generaciones y territorios, la estructura tributaria, el financiamiento de las redes de seguridad social y las políticas de empleo y seguridad, entre otras.

4.3 Productividad. Siendo las personas la piedra angular del desarrollo, es fundamental asegurar que participen plenamente en el proceso de generación de ingresos con trabajo decente. El aumento sostenido de la productividad es el punto de encuentro de las inversiones realizadas para mejorar su salud, conocimientos y destrezas, y el uso de esas capacidades en el mundo laboral.

4.4 Equidad. Las personas deben tener acceso a la igualdad de oportunidades para desplegar sus potencialidades y participar equitativamente en todas las esferas de la 
sociedad. Particular importancia tiene en este marco la equidad de género no sólo por razones de justicia social, sino también porque su plena incorporación este volvería más fácil el esfuerzo del desarrollo.

2.5 Ciudadanía plena. En un régimen auténticamente democrático la gente debe participar en los diversos procesos que afectan sus vidas y asegurarse de que sus intereses estén plenamente representados en las decisiones políticas.

\section{Visión y objetivos de largo plazo}

\subsection{Visión}

Ser un país de alto desarrollo humano al cabo de una generación.

\subsection{Objetivos de largo plazo}

- Lograr que todas las personas en edad de trabajar y con voluntad de hacerlo tengan trabajo decente. Dentro de la estrategia que se plantea, este objetivo es crucial para el logro de los demás y consiste en disminuir de manera progresiva el desempleo, el subempleo y el empleo informal hasta llegar al pleno empleo.

- Cobertura universal en los siguientes pilares de la política social. Familia e hijos (atención prenatal, cuidado y educación temprana); educación (bachillerato y opciones para educación superior); salud (acceso con calidad a redes públicas o privadas); empleo (capacitación, reconversión laboral y seguro por desempleo); vivienda e inversiones (propiedad de la vivienda); pensiones y discapacidad.
- Corregir los desbalances macroeconómicos y financieros internos y externos. Concretamente se requiere pasar de una economía de consumo a una economía de producción, lograr un equilibrio ahorroinversión a un nivel creciente de ambas variables, equilibrar las finanzas públicas y reorientarlas a favor del desarrollo y revertir el desequilibrio externo mediante el mejoramiento sostenido de la productividad y la competitividad de la economía.

- Reactivar la economía y sentar las bases para el logro de un crecimiento robusto y sostenido. El crecimiento económico estaría fundamentado en el fortalecimiento de la relación ahorro-inversión y el aumento de la productividad laboral. Sobre la base de estos ejes se promovería el desarrollo de cadenas de valor, la diversificación de la producción y un mejoramiento de la competitividad que permita reducir progresivamente la brecha comercial, tanto por la vía de aumentar exportaciones como de sustituir importaciones.

\section{La estrategia}

La estrategia consiste en la construcción de un círculo virtuoso que eleve los niveles de ahorro, inversión, competitividad, crecimiento y empleo (ver gráfico 1), y que a la vez permita construir una relación armónica entre el desempeño macroeconómico del país y el mejoramiento progresivo del nivel de bienestar de la población.

El punto de partida de la estrategia es el aumento de la tasa de ahorro nacional a partir de la introducción de nuevas contribuciones obligatorias vinculadas a la ampliación progresiva de losámbitos cubiertos por las redes de seguridad social, más algunas medidas fiscales que permitan modificar 
el mapa de incentivos a favor de la inversión y la producción y en detrimento del consumo y las importaciones. Se trata, por lo tanto, de sacrificios que tendrán que hacer todos los miembros de la sociedad (gobierno, empresarios y familias) que tomarán la forma de menor consumo presente, pero que tendrán como contrapartida mayores niveles de inversión en productividad y un mejoramiento de la competitividad de la economía. Una de las ventajas es que es posible hacer sacrificios. El PIB per cápita y el ingreso per cápita de El Salvador hoy, superan a los niveles alcanzados en 1990 en $63 \%$ y $71 \%$, respectivamente.

Por otra parte, el crecimiento del país no puede continuar dependiendo predominantemente de la expansión del consumo, por lo que es necesario y posible aumentar el ahorro y desalentar el consumismo. Esta propuesta contiene políticas concretas para lograrlo.

Los sacrificios al consumo presente, que significa el ahorro, habrán valido la pena si los recursos se invierten debidamente. El uso que se define para el ahorro en esta estrategia es la inversión en capacidades de las personas y la construcción de infraestructura que ayude a mejorar los niveles de empleo y competitividad; esto es, inversión en productividad. Ello significa, además, que se descarta la utilización de los recursos del ahorro para financiar gasto corriente, incluidos los subsidios al consumo. Esto, por dos razones: en primer lugar, porque la estrategia busca romper con la tendencia actual hacia un mayor endeudamiento público y privado, que tendrá que ser pagado por las futuras generaciones en la medida que se continúen postergando los ajustes. En segundo lugar, porque únicamente destinando los recursos a inversiones productivas se podrá generar el retorno necesario para seguir retroalimentando el círculo virtuoso que se busca construir.
De esta forma, los primeros dos componentes del círculo: ahorro e inversión, resumen la manera en que se ha previsto financiar las crecientes inversiones destinadas a expandir las capacidades de las personas y mejorar la infraestructura.

El tercer elemento del círculo es la competitividad. El impacto de los mayores niveles de inversión social y en infraestructura puede limitarse, afectando la competitividad delaeconomía, silosmercadosnofuncionanadecuadamente y si no se dispone de un clima apropiado de inversiones. En este Informe se ha documentado la persistencia de fallas en mercados clave de la economía salvadoreña y es por ello que la estrategia incluye un fortalecimiento de la institucionalidad para contar con mercados favorables a la gente. De igual manera, se ha destacado la importancia que tiene para el éxito de cualquier estrategia la reducción de los niveles de inseguridad y violencia, así como una mejor gestión de los diferentes riesgos a los que está sometido el país

El cuarto elemento es el crecimiento económico, que en este caso estaría fundamentado en la reducción de los costos de producir en el país, que resultaría de la mayor productividad laboral, la ampliación de la infraestructura y el mejoramiento de la institucionalidad.

Finalmente, el quinto elemento es el empleo. Para que se pueda consolidar y retroalimentar un círculo virtuoso, el tipo de empleo que se debe promover es, en una primera etapa, aquel que está integrado a las redes de seguridad social. Incluso, se propone que se utilice el porcentaje de la PEA, que goza de los beneficios de las redes de seguridad social como la vara para medir los resultados de la estrategia en el corto y el mediano plazo. Esto, porque el círculo se fortalece a medida que dicho porcentaje aumenta y porque se trata de una modalidad de inserción laboral próxima al trabajo decente. Para obtener éxito en la generación de empleo 
se requiere que se aprovechen las mayores capacidades desarrolladas por las personas para generar valor mediante nuevas actividades económicas y la expansión de otras. Aquí adquiere importancia la transformación del sistema de incentivos económicos a favor de aquellas actividades en las que el país tenga mayores potencialidades de desarrollar ventajas competitivas y que sean, además, intensivas en la mano de obra de que se disponga en cada etapa (apuestas estratégicas). De esta manera, el empleo se convierte en el punto de encuentro entre las políticas que tradicionalmente se conciben en forma separada, ya sea como sociales o como económicas.

En el diagrama 1 se detalla el círculo virtuoso que se pretende construir con la estrategia:

\section{Diagrama 1. Compatibilizando la política económica y la política social}


Este nuevo enfoque también reconoce que una buena política de pensiones comienza con el cuidado de los bebés. Esto significa que la mejor manera de procurar el bienestar de las personas es realizando oportunamente las inversiones que le aseguren la posibilidad de tener una vida plena en sus diferentes etapas (antes, durante y después del ingreso al mercado laboral) (ver diagrama 2).

En términos generales, es en la infancia y la adolescencia cuandoserealizanelgrueso delas inversionesen capacidades y la formación de la persona en valores de convivencia y respeto. Sus fines son: facilitar la exitosa inserción de las personas en la siguiente fase del ciclo (mercado laboral) y cultivar un sentido de pertenencia y valores ciudadanos. Este segundo momento debe caracterizarse, principalmente, por el despliegue de las capacidades de las personas en el mercado laboral y por altos niveles de participación en los procesos que afectan sus vidas (ejercicio de la ciudadanía). En esta fase debe intensificarse el ahorro con el objetivo de financiar la formación de las siguientes generaciones, la inversión en la adquisición de activos y asegurar un nivel de ahorro e inversión adecuado para la edad de retiro.

Las inversiones que se realizaron en la primera fase rendirán mayores frutos en la medida que las personas puedan crear valor a partir de sus conocimientos, habilidades y destrezas; esto es, en la medida que se incorporen adecuadamente al mercado laboral y tengan un entorno donde prevalezcan el respeto a sus opciones de vida y a sus derechos fundamentales. Visto así, en la medida que muchas personas emigren del país o no formen parte de la fuerza laboral, las inversiones que se hayan realizado en ellas, en parte, se perderán.

\section{Diagrama 2. Política social y etapas del ciclo de vida}

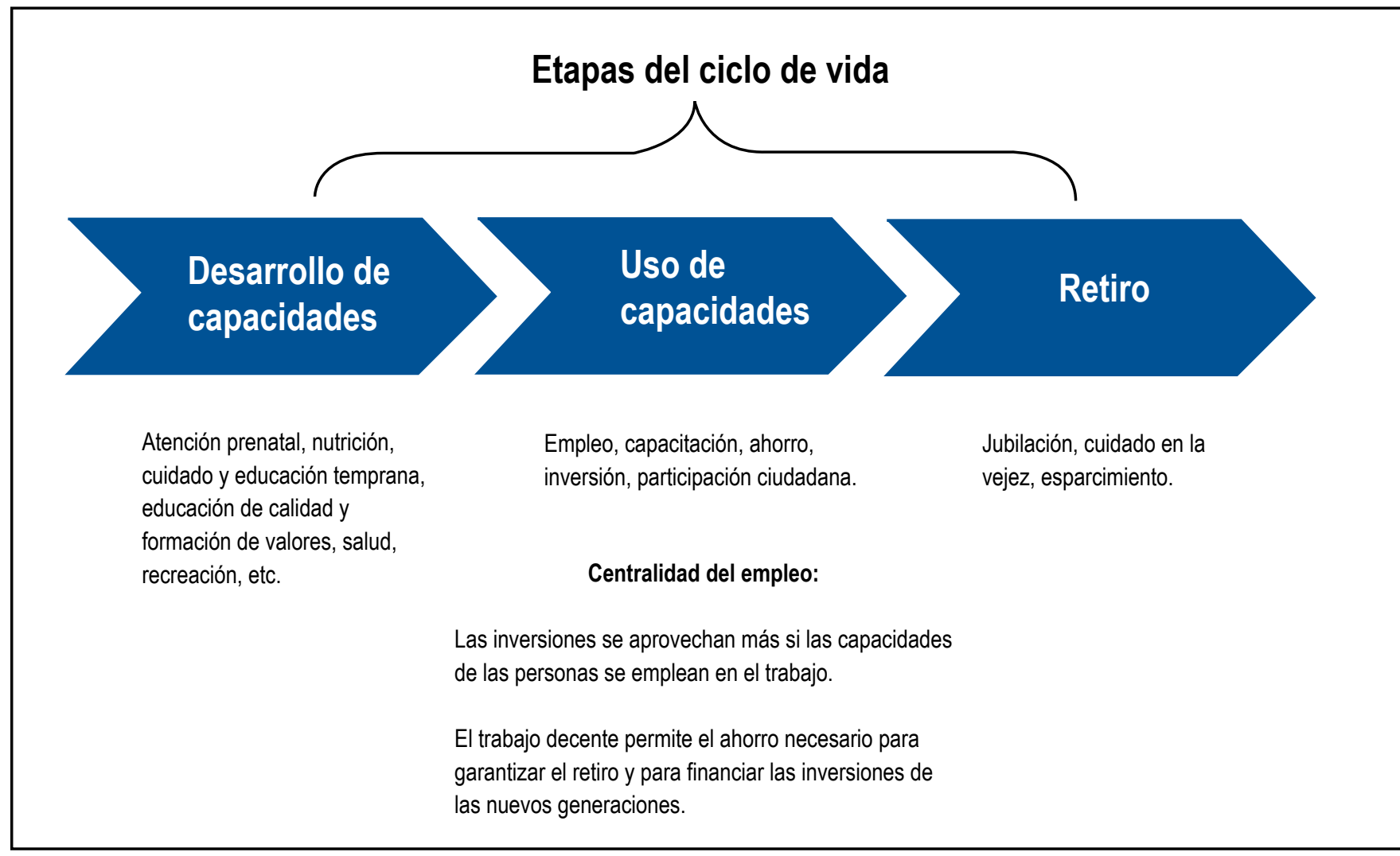


Como resultado de esta conceptualización e intervenciones prácticas, se espera que el país logre colocarse entre las sociedades con mayores niveles de bienestar, progreso económico y niveles de competitividad.

Dados los bajos niveles de ahorro y la alta vulnerabilidad a la que están expuestas muchas familias y personas, es necesario apoyarlas a planificar su futuro.

Para tal efecto, se propone la creación del fondo probienestar de la economía familiar como una respuesta práctica a estos desafíos. Este Fondo se crearía a partir de aportaciones de empleados y empleadores en cuentas de ahorro, con dos fines: favorecer la economía familiar y el pleno desarrollo de las familias, y servir como una fuente de financiamiento de inversiones productivas que aseguren un rendimiento mínimo para cada miembro.

Desde el punto de vista de los miembros del Fondo, se trata de un ahorro programado que permitirá financiar los pilares de la política social, a saber: el cuidado de sus hijos en edades tempranas, su educación en etapas superiores, así como la propia capacitación, reconversión laboral y compensación en caso de desempleo. También, será el medio para financiar los gastos de salud de su núcleo familiar y la manera de asegurar que con el paso de los años tendrá capacidad para ser propietario de su vivienda y tener ingresos suficientes en la vejez.

\subsection{1 ¿Quiénes aportarían al Fondo?}

En primera instancia, en el Fondo ahorrarán todas las personas integradas a las redes de seguridad social. La apuesta es incorporar a dicho fondo a la totalidad de personas que participan el mercado laboral, independientemente de si están en el sector formal o informal. Esto se logrará en la medida que el Fondo sea una alternativa de ahorro atractiva para todos los ciudadanos.

\subsubsection{Un ahorro programado a lo largo del ciclo de vida}

Cada miembro del Fondo poseerá una cuenta general de capitalización individual alimentada por las contribuciones de empleadores y trabajadores. De esa contribución general se acreditan con diferente proporción cada una de las cuentas del miembro (diagrama 3). La lógica de acreditación de las cuentas está vinculada al ciclo de vida de las personas. ${ }^{1}$

\subsubsection{Las cuentas que componen el Fondo}

La cuenta de red del cuidado tiene como objetivo central contar con los recursos necesarios para crear una red de calidad que atienda el desarrollo de los niños en sus etapas más tempranas de la vida; y de esta forma permitir que ingresen al mercado las personas que ahora no pueden hacerlo debido a que están a cargo de tareas de su cuidado. Esto además facilitará la ampliación de la red de cuidado como sector formal y cadena de valor en la economía.

La cuenta de vivienda e inversiones está destinada a financiar parte o la totalidad de la vivienda del miembro del Fondo. El objetivo es crear un país de propietarios. Sin embargo, es necesario establecer el mínimo que deben cubrir los ahorros para que el miembro del Fondo pueda hacer uso de estos, ya sea para compra de vivienda o utilizarlos en otro tipo de inversiones.

La cuenta de educación tiene como objetivo guardar fondos que sirvan para financiar la educación postsecundaria de los hijos del miembro de la cuenta.

\footnotetext{
${ }^{1}$ En general, una persona más joven verá acreditada una mayor proporción en la cuenta dedicada a la vivienda e inversiones que una persona de las cohortes más cercanas al retiro, donde se privilegian los recursos destinados a la cuenta para la vejez.
} 


\section{Diagrama 3. Cobertura de la cuenta de ahorro de miembros del Fondo}

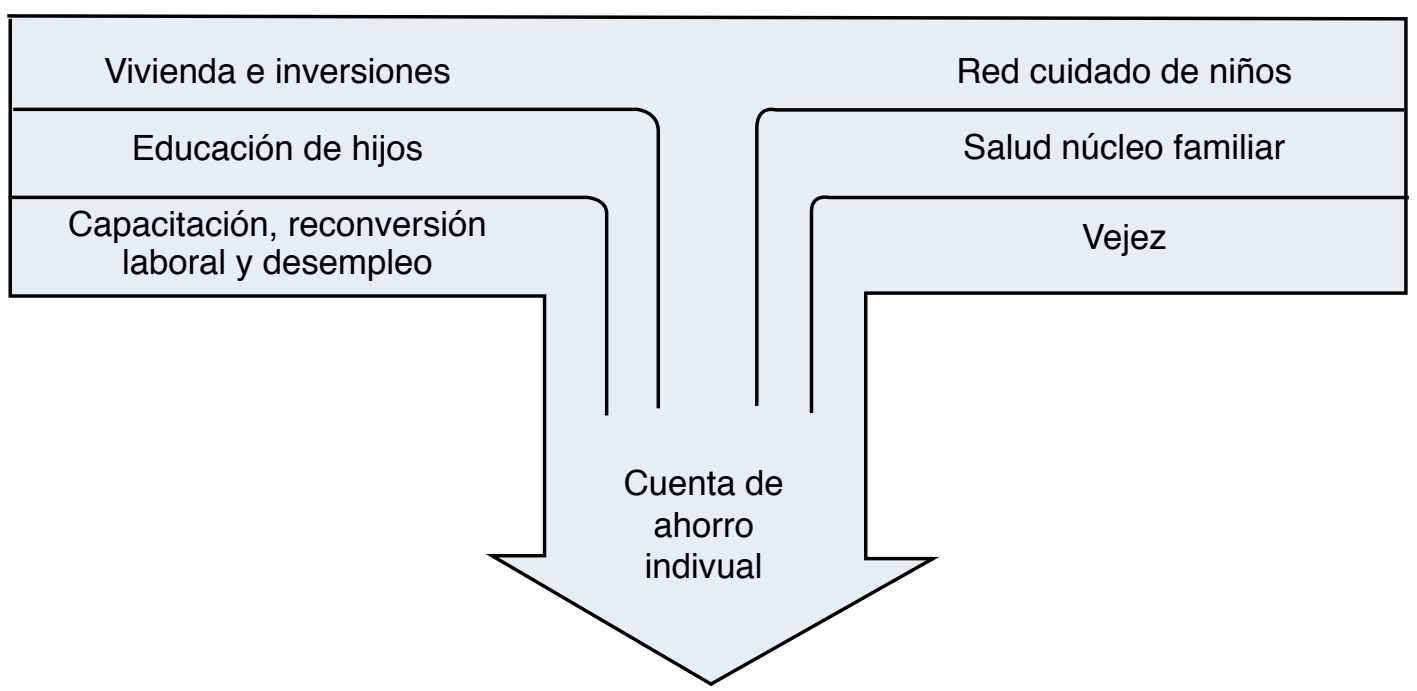

La cuenta de salud contribuye al ahorro de cierto monto de dinero para sufragar los gastos sanitarios del miembro y sus familiares, y para financiar un seguro médico en caso de enfermedades catastróficas del titular.

Otro de los ahorros programados es el fondo destinado a financiar cursos de capacitación o reconversión laboral. El objetivo de este mecanismo es evitar que las personas que pierdan su empleo caigan en subempleo, y reducir al mínimo el tiempo de permanencia fuera del mercado laboral. Esta cuenta también puede financiar un seguro por desempleo.

La cuenta de vejez permite contar con los fondos necesarios para un retiro digno. Esta cuenta se alimenta a lo largo del período laboral de las personas y con los excedentes de las cuentas anteriores.

Los recursos remanentes, en cualquiera de las cuentas, son trasladados a la cuenta de vejez al momento del retiro. De tal manera que, si una persona al momento de hacerse parte del Fondo ya contaba con su vivienda y no hace uso de los fondos de su cuenta de vivienda e inversiones, estos recursos automáticamente son dirigidos a la cuenta de vejez, o a la de educación.

\subsubsection{Origen, etapas y características del Fondo}

La creación de este Fondo se debe llevar a cabo por etapas. La primera debe estar dedicada a activar las cuentas de ahorro destinadas a la red del cuidado, la educación y el financiamiento de vivienda e inversiones. La segunda fase debe compatibilizar los aportes que realizan las personas al ISSS, a Insaforp y al sistema de pensiones, de manera que se logren incorporar estos recursos en la cuenta general de ahorro (diagrama 3).

Se sugiere que el Fondo parta de una contribución del 2,5\% del salario de los trabajadores, complementado con un aporte de igual porcentaje por parte de los empleadores. ${ }^{2}$ Es importante que la tasa de contribución se incremente a medida que vayan aumentando los salarios reales de las personas.

\footnotetext{
2 En principio, la mayoría de estos recursos serán acreditados a la cuenta relacionada con vivienda e inversiones, además de canalizar los recursos restantes hacia la cuenta de red del cuidado y la de educación.
} 


\subsubsection{La orientación estratégica del Fondo}

El Fondo deberá contar con una junta directiva que tome las decisiones que garanticen el uso eficiente de los recursos y protejan el valor de los ahorros. Se propone que esta junta directiva cuente con representantes del Gobierno, empresarios y trabajadores, así como de personalidades cuyas experiencia, capacidades y honorabilidad sirvan de garantía para la toma de decisiones. De forma similar a como ocurre en otros países, las cuentas del Fondo deben ganar un interés mínimo garantizado por el Estado. Ello asegura que los recursos serán utilizados exclusivamente en proyectos con un retorno económico y que el Fondo constituya una opción atractiva en el mercado.

\subsection{Aumento de la inversión social}

El Fondo pro-bienestar de la economía familiar es la gran apuesta para que el país establezca a largo plazo un piso social universal y adquiera capacidad para financiar con recursos propios el círculo virtuoso de desarrollo humano (diagrama 1). En el corto y el mediano plazo, sin embargo, será necesario liberar un porcentaje creciente del presupuesto del Estado para realizar las inversiones requeridas para reducir los déficit sociales acumulados y sentar las bases del nuevo modelo de desarrollo.

Estimaciones realizadas por el PNUD indican que, para asegurar el acceso universal a servicios sociales básicos tales como nutrición infantil, salud primaria, educación secundaria y vivienda digna, El Salvador debe de aumentar la inversión social en alrededor de 4,3\% del PIB durante los próximos años.

Solo la puesta en marcha del nuevo sistema integrado de salud impulsado por la presente administración requerirá de un aumento de recursos fiscales equivalentes a cerca del $1 \%$ del PIB. A ello habría que sumar los mayores requerimientos en el área de educación, capacitación, ciencia y tecnología, estimados en alrededor de un 3\% del PIB adicional.

Para armar el ciclo virtuoso no solo se requieren más recursos, sino también su mejor aprovechamiento. Algunas recomendaciones nuevas, o ya contenidas en informes y estudios anteriores, son las siguientes:

a. Universalización del dominio de la computación y de una segunda lengua (preferentemente el inglés). Este objetivo debe alcanzarse al egresar del bachillerato. Estimaciones realizadas para este informe indican que los recursos requeridos anualmente para alcanzarlo son inferiores a los que se destinan actualmente para subsidiar el gas propano o para zapatos, uniformes y útiles escolares.

b. Redefinir tanto el horario educativo como el año escolar. El Salvador también necesita que los estudiantes de educación primaria y media vuelvan al horario de por lo menos 7 horas diarias. Esto permitirá disponer del tiempo requerido para universalizar la enseñanza de computación y la segunda lengua, además de que reduciría los riegos de abandono y reclutamiento por las pandillas de niños, adolescentes y jóvenes.

c. Mejoramiento de la calidad de la educación, Para ello lo fundamental es la política de reclutamiento, evaluación e incentivos hacia el personal docente y los contenidos de la currícula educativa.

d. Tecnología. La tecnología debe convertirse en el pilar fundamental del desarrollo económico. El Salvador debe participar en esta "revolución electrónica" más intensiva en capital humano que en capital físico, y en donde el talento, el conocimiento y la imaginación 
cuentan tanto o más que las instalaciones industriales o los recursos naturales.

\subsection{Apuestas estratégicas}

La gran apuesta estratégica de esta propuesta es alcanzar el bienestar de la gente a través del ahorro y la inversión social, para el desarrollo de sus capacidades. Esta apuesta central debe ir acompañada de medidas económicas que generen oportunidades para el pleno uso de esas capacidades.

El Salvador necesita pensar y definir cuáles industrias serán el motor de su desarrollo económico. Estas actividades serán también las que determinen los beneficios que puedan obtenerse de la integración a los mercados internacionales, a través de la habilidad que tenga el país para posicionarse con más productos de calidad en el mercado mundial. Las apuestas estratégicas para creación de empleo en el corto y mediano plazo deben de estar en armonía con sus objetivos y metas de mediano y largo plazo y con las fortalezas y limitaciones presentes. Considerando que el objetivo último es incrementar el empleo integrado a redes de seguridad social, las apuestas estratégicas deben alinearse a la creación masiva de empleos en el país.

En un primer momento, las apuestas estratégicas deben tener capacidad de absorber la mano de obra que existe en el país, la cual en su mayoría es no calificada. En concordancia, una apuesta estratégica podría ser el sector construcción, tanto de vivienda como de infraestructura clave para la mejora de la competitividad. Asimismo, podría apostarse por una expansión en la agroindustria y las actividades de exportación de productos étnicos, que cuentan con un mercado cautivo con enorme potencial. También podría fomentarse la actividad turística, utilizando para ello un programa de fomento como el que se describe más adelante.
Luego, las apuestas estratégicas deberán orientarse a sustentar el círculo virtuoso de ahorro, inversión en capacidades, incremento en la competitividad, crecimiento y generación de empleos. Es así que puede pensarse en apuestas estratégicas tales como la maquila de productos de mayor sofisticación y de servicios profesionales (call centers, servicios de contabilidad, legales, etc.) y convertir al país en un centro regional de servicios logísticos.

\subsection{Fortalecimiento institucional}

En La riqueza de las naciones, Adam Smith advertía que el comercio y la manufactura difícilmente pueden florecer en un Estado en el que no exista cierto nivel de confianza en la justicia del Gobierno. Y es que la economía está incluida en una estructura de leyes e instituciones que deben ser tomadas en cuenta explícitamente en el diseño de las políticas. No basta con el desarrollo espontáneo de los mercados para asegurar que se resolverán los problemas económicos; por el contrario, es posible mejorar de manera deliberada el orden económico.

Algunos los llaman "reglas del juego", otros "arreglos institucionales", Lo cierto es que, pese a que hoy en día se reconoce ampliamente la relevancia del marco institucional, el país ha carecido de una agenda que busque fortalecer la calidad de las instituciones con las que se cuenta. Y esto puede deberse a que en muchas ocasiones el debate institucional se ha entendido erróneamente como una discusión entre más o menos Estado, sin reconocer el papel complementario de las instituciones para el buen funcionamiento de los mercados. Los mercados que no funcionan bien están caracterizados por una serie de limitaciones para la adecuada asignación de recursos y por niveles subóptimos de eficiencia. Por esta razón, en la práctica, las economías de mercado exitosas son vigiladas por una amplia gama de instituciones que regulan las 
conductas en diversos sectores. No es coincidencia que Estados Unidos - uno de los mercados más libres- sea el que con más dureza aplica su legislación pro-competencia. En El Salvador, la poca competencia que prevalece en el mercado de ciertos sectores considerados estratégicos para la economía (e.g. sistema financiero, energía eléctrica, transporte, telecomunicaciones, hidrocarburos) vuelve imperativa la necesidad de contar con un marco legal que proteja y promueva la libre competencia. También son necesarios los entes que, con suficiente autonomía, regulen y supervisen esos mercados. Sin esta característica esas instancias difícilmente podrán ejercer eficazmente su función de supervisión y regulación, no habrá transparencia, se generarán conflictos de interés y actividades de captación de rentas, y no se formarán cuadros técnicos independientes que cumplan adecuadamente con las funciones encomendadas.

Además, el país necesita adoptar con urgencia un sistema de contrataciones en la administración pública de carácter meritocrático (un número limitado de puestos pueden seguir designándose a personal de confianza), establecer un sistema de incentivos y remuneración basado en desempeño que estimule la productividad en el trabajo, definir una política que permita balancear los objetivos de flexibilidad y estabilidad en los puestos. Como primer paso, se recomienda que se cree una entidad con suficiente independencia, que tenga a su cargo la reforma integral al servicio civil.

Para elevar la eficiencia y eficacia en la gestión del Estado, también se recomienda abandonar el enfoque incrementalista de asignación del presupuesto por una gestión basada en resultados, para lo cual es necesario modificar los criterios tradicionales de funcionamiento de las instituciones y de la forma en que se administran los recursos. Concretamente, implica un cambio en el proceso de elaboración de los presupuestos, privilegiando el logro de resultados por encima de una asignación basada en insumos (por ejemplo, personal, compra de bienes y servicios, gastos varios, etc.)

Finalmente, es necesario fortalecer la transparencia y rendición de cuentas y el control de la corrupción mediante un código de ética y la dotación de autonomía e independencia a la Corte de Cuentas de la República. 